\title{
DETECTION OF HEMATOPOIETIC STEM CELLS IN MICROSCOPY IMAGES USING A BANK OF RING FILTERS
}

\author{
Sungeun Eom, Ryoma Bise, Takeo Kanade \\ The Robotics Institute, Carnegie Mellon University \\ 5000 Forbes Avenue, Pittsburgh, PA 15213
}

\begin{abstract}
We present a method for robustly detecting hematopoietic stem cells (HSCs) in phase contrast microscopy images. HSCs appear to be easy to detect since they typically appear as round objects. However, when HSCs are touching and overlapping, showing the variations in shape and appearance, standard pattern detection methods, such as Hough transform and correlation, do not perform well. The proposed method exploits the output pattern of a ring filter bank applied to the input image, which consists of a series of matched filters with multiple-radius ring-shaped templates. By modeling the profile of each filter response as a quadratic surface, we explore the variations of peak curvatures and peak values of the filter responses when the ring radius varies. The method is validated on thousands of phase contrast microscopy images with different acquisition settings, achieving $96.5 \%$ precision and $94.4 \%$ recall.
\end{abstract}

Index Terms - Cell detection, ring filter, quadratic form, hematopoietic stem cells

\section{INTRODUCTION}

Hematopoietic stem cells (HSCs) are blood-forming stem cells that give rise to all the blood and immune cell types, including myeloid and lymphoid lineages. They are one of the only stem cell types that are routinely used in therapies today. HSCs were also discovered to be able to form other kinds of cells, such as muscle, blood vessels, bone, and liver cells [1]. Once confirmed, it may eventually be possible to use HSCs to regenerate a wide array of cells and tissues.

Two fundamental barriers hinder the development of new and improved HSC-based treatments: 1) the lack of costeffective strategies to boost the number of HSCs in vitro in order to meet clinical and research demands; and 2) the lack of understanding of the condition and mechanism for controlled differentiation of HSCs into various types of tissues. To overcome these barriers, novel computational toolsets are required to automatically and reliably quantify the numbers and positions of HSCs in population environments, as they divide into daughter cells. Since phase contrast microscopy

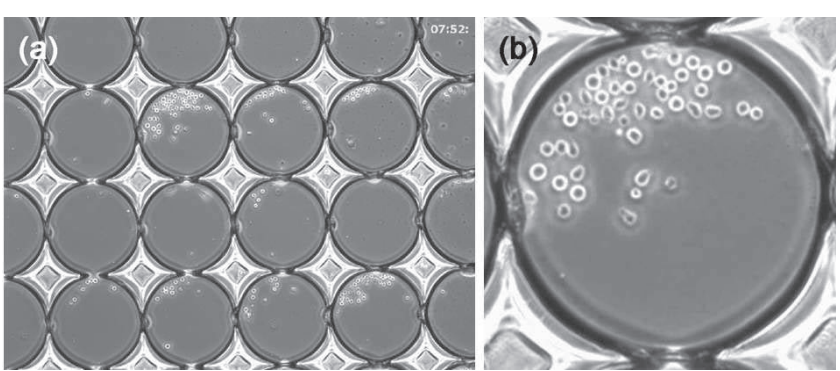

Fig. 1. HSCs imaged with phase contrast microscopy. (a) HSC populations in a microwell array. (b) HSC population confined in a single microwell.

is the primary imaging technique for the long-term monitoring of HSCs in vitro, an accurate and robust method for the detection of HSCs in phase contrast microscopy images is an important prerequisite for implementing such toolsets.

Under a phase contrast microscope, HSCs typically appear as round objects surrounded by bright halos (Fig. 1). There are two standard methods that can be applied to detect such objects: Hough transform and correlation. Hough transform based detection methods [2,3,4], which use image gradient or edge information, have some drawbacks in our case due to the variations of cell shape and appearance. The variations cause low peaks in an output accumulator space and thus lead to false negatives or inaccurate detection (Fig. 2a). Correlation based methods $[5,6,7,8]$ partly overcome those drawbacks by using cell image templates, but still there are problems (Fig. 2b). In particular, false positives tend to occur on the background surrounded by cells because neighboring bright halos contribute to high responses outside the cells. As the number of cells increases the false positives would appear more often.

Based on the observations, we propose a simple and effective method that utilizes the contextual information provided by multiple filter outputs. The method examines only the changes of peak curvatures and peak values of the filter outputs to confirm true cells. Our proposed method has been extensively validated in thousands of phase contrast microscopy images, and was shown to consistently outperform the standard methods. 


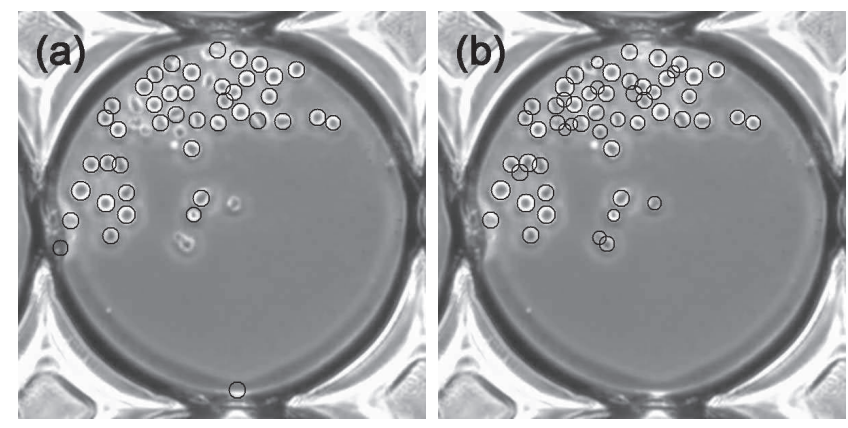

Fig. 2. Detection using the standard methods: (a) Hough transform. (b) correlation.

\section{CELL DETECTION}

Our cell detection scheme consists of three steps: 1) filter the input image with multiple-radius ring filters; 2) locate cell candidate locations and radii by detecting and thresholding local maxima of the ring filter outputs, allowing a relatively high false positive rate; and 3) eliminate false positives by examining the output pattern of the multiple filters

\subsection{Cell Candidates}

We model the shape and appearance of an HSC by a ring pattern as depicted in Fig. 4a. The pattern consists of a bright ring on a dark background. The radius of the ring, denoted $r_{c}$, is defined as the radius of its outer circle. The width of the ring, denoted $w$, equals the difference between the radii of its outer and inner circles. The corresponding ring filter template has an identical profile as the pattern. Since the radius of cells changes within some range as they proliferate, we use multiple ring filters corresponding to the range.

When the filters are correlated with an image containing ring patterns, the outputs have peaks (local maxima) at positions where each filter is matched to the patterns with the same size. Cell candidate locations are obtained by detecting local maxima with values exceeding a preset threshold in the three-dimensional ring filter output space; the first two dimensions are spatial, and the third is the dimension of radius, which we call the contextual dimension. The threshold is set to be low to achieve a near-zero false negative rate, which ensures most (if not all) cells are detected. An example of detected cell candidates is shown in Fig. 3.

\subsection{False Positive Elimination}

There are two types of false positives, as can be seen in Fig. $3 \mathrm{~b}$. The first type is due to multiple local maxima for a single cell that are generated by sightly different filters in size, and the second one that appears in the background is caused by bright halos of neighboring cells.
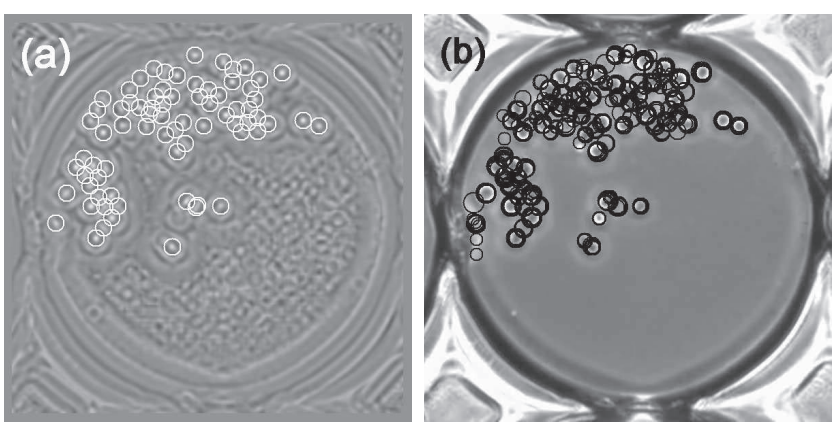

Fig. 3. Cell candidates. (a) Output of ring filter with radius of 12 pixels and the corresponding candidates. (b) All candidates from ring filters with radii between 9 and 15 pixels.

The first type of false positives can be eliminated by extending the idea of standard matched filtering. As illustrated in Fig. 4, the highest peak occurs when the radius of the filter matches exactly to that of the pattern, and lower peaks are generated when the filter mismatches slightly with the pattern. The phenomena suggest that to detect the presence of a ring pattern of radius $r_{c}$ (and not of any other radii), exactly three ring filters are needed: one with radius $r_{c}$, one with a radius smaller than $r_{c}$, and another with a radius larger than $r_{c}$.

Specifically, we utilize a ring filter bank consisting of three ring filters with radii $r_{c}-1, r_{c}$, and $r_{c}+1$ to detect a cell with radius $r_{c}$. Using these three filters, the detection of a ring pattern amounts to locating local maxima that are higher than the peak values at radii $r_{c} \pm 1$. Since we already have multiple filter outputs corresponding to a range of cell radii, we only need to check the peak curvatures (local maxima) and compare the peak values at positions of cell candidates in the three-dimensional filter output space.

(a)

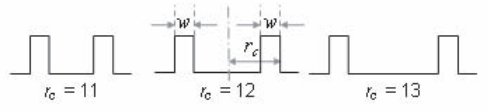

(b)

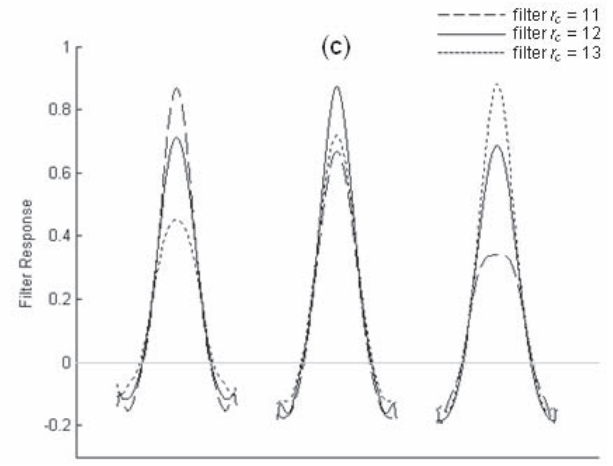

Fig. 4. Output variations of a multiple-radius ring filter bank. (a) Input ring patterns with different radii. (b) A multipleradius ring filter bank. (c) The outputs of the filter bank applied to each ring pattern. 
In order to robustly extract the peak curvatures and values at any location, we fit a quadratic surface model to the filter output in a fitting window of $M \times M$ pixels around the specified location. The peak position and value are then computed from the fitted quadratic surface. Specifically, the values of the filter output in a fitting window can be approximated by a quadratic polynomial surface given by,

$$
g(x, y)=a_{0}+a_{1} x+a_{2} y+a_{3} x y+a_{4} x^{2}+a_{5} y^{2} .
$$

The peak position, denoted $\left(x_{p}, y_{p}\right)$, can be computed as:

$$
x_{p}=\frac{a_{2} a_{3}-2 a_{1} a_{5}}{4 a_{4} a_{5}-a_{3}^{2}}, \quad y_{p}=\frac{a_{1} a_{3}-2 a_{2} a_{4}}{4 a_{4} a_{5}-a_{3}^{2}},
$$

which can be derived by setting the partial derivatives of $g(x, y)$ with respect to $x$ and $y$ to zero. The principle curvatures are given by the eigenvalues of the matrix:

$$
\left(\begin{array}{cc}
a_{4} & \frac{1}{2} a_{3} \\
\frac{1}{2} a_{3} & a_{5}
\end{array}\right)
$$

The polynomial coefficients $\left\{a_{0}, \ldots, a_{5}\right\}$ are determined by computing the least squares solution for

$$
\left(\begin{array}{c}
f_{1} \\
\vdots \\
f_{i} \\
\vdots \\
f_{n}
\end{array}\right)=\left(\begin{array}{cccccc}
1 & x_{1} & y_{1} & x_{1} y_{1} & x_{1}^{2} & y_{1}^{2} \\
\vdots & \vdots & \vdots & \vdots & \vdots & \vdots \\
1 & x_{i} & y_{i} & x_{i} y_{i} & x_{i}^{2} & y_{1}^{2} \\
\vdots & \vdots & \vdots & \vdots & \vdots & \vdots \\
1 & x_{n} & y_{n} & x_{n} y_{n} & x_{n}^{2} & y_{1}^{2}
\end{array}\right)\left(\begin{array}{c}
a_{0} \\
a_{1} \\
a_{2} \\
a_{3} \\
a_{4} \\
a_{5}
\end{array}\right),
$$

where $f_{i}=f\left(x_{i}, y_{i}\right)$ is the value of a pixel in the fitting window, and $n$ is the total number of pixels in the window. This over-determined linear system can be expressed as $\vec{f}=\mathbf{A} \vec{a}$, which has a closed-form solution $\vec{a}=\left(\mathbf{A}^{T} \mathbf{A}\right)^{-1} \mathbf{A}^{T} \vec{f}$.

In the same framework, the second type of false positives is removed by incorporating roundness into filter outputs. As mentioned earlier, the false positives appear around cells and thus happen more often as the number of cells increases. It is because the ring template is not able to distinguish whether it is matched at the inside or outside of cells. As long as the halos of cells lie on the ring area of the template, the filter produces similar output. In this case the only difference appears in the roundness of edges that are formed around a cell candidate.

We first extract edges in the image areas containing cell candidates, and then find edge segments for each cell candidate. Since the cells are round the edge segments expect to have positive curvatures for a true cell. Therefore the following curvature-based roundness can be used as a weight of filter outputs to discriminate the false positives from true cells:

$$
R=\frac{\sum_{i=1}^{N} L\left(e_{p o s}^{i}\right)}{2 \pi r_{c}}
$$

where the numerator is the sum of the length of edge segments $e^{i}$ with positive curvature and it is normalized by the length of a circle with radius $r_{c}$ of a cell candidate.

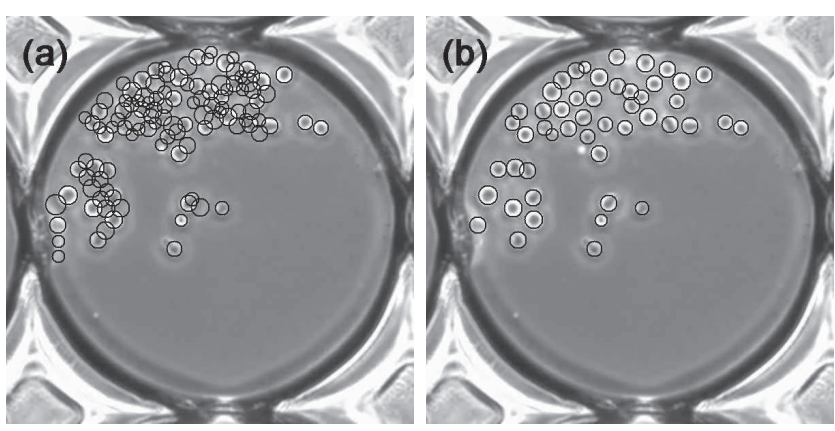

Fig. 5. False positive elimination from cell candidates. (a) Duplicate removal. (b) Final detection result.

\section{CELL CULTURE AND IMAGING}

Bone marrow CD34+ HSCs (Stem Cell Technologies, Vancouver, BC, Canada) were cultured in serum-free expansion media with Stem Span Cocktail CC100 (Stem Cell Technologies, Vancouver, BC, Canada) at $37^{\circ} \mathrm{C}, 5 \% \mathrm{CO}_{2}$. The cells were seeded in microwells at a density of $1-5$ cells/well. Time-lapse images were taken on 1-minute intervals for 5 days using a Carl Zeiss Axiovert 200M microscope with an EC-Plan Neofluar $5 \times$ phase objective (Carl Zeiss Microimaging, Thornwood, NY), a heated stage incubator at $37^{\circ} \mathrm{C}, 5 \% \mathrm{CO}_{2}$, and $90 \%$ humidity (Pathology Devices Inc., Westminister, MD), and AxioVision 4.7.1 software.

\section{EXPERIMENTAL RESULTS}

We conducted experiments on two phase contrast microscopy image sequences of HSCs from two different cell cultures. The images were extracted from the original $1388 \times 1040$ pixel images showing a $4 \times 3$ array of microwells to contain only one microwell, as shown in Fig. 1. There are two reasons for using the low magnification images. First, more wells in the field of view means more chances to observe more experiments per session. Second, the halo effect for the cells appears more prominent compared to size, which makes the cells more spherical in appearance.

We compared the performance of the proposed detection method with the standard methods: Hough transform [2] and correlation [5]. In our method, we utilized ring filters with radii between 8 and 16 pixels since the radii of cells range between 9 and 15 pixels in our experiments and two more filters are required to detect the smallest and the largest cells with the filter bank. For all the methods the threshold was set to $20 \%$ of the maximum value of ring filter outputs and accumulation array in each frame, respectively. Based on the ground truth created by manual counting, the overall performance is presented in terms of precision $\mathrm{TP} /(\mathrm{TP}+\mathrm{FP})$ and recall $\mathrm{TP} /(\mathrm{TP}+\mathrm{FN})$, where $\mathrm{TP}, \mathrm{FP}$, and $\mathrm{FN}$ denote the number of true positives, false positives, and false negatives, respectively. 
Table 1. Comparison of detection performance.

\begin{tabular}{lccc}
\hline Method & $\begin{array}{c}\text { Hough } \\
\text { Transform }\end{array}$ & CORRELATiOn & $\begin{array}{c}\text { OUR } \\
\text { METHOD }\end{array}$ \\
\hline Precision & $91.2 \%$ & $85.7 \%$ & $96.5 \%$ \\
Recall & $86.5 \%$ & $90.9 \%$ & $94.4 \%$ \\
\hline
\end{tabular}

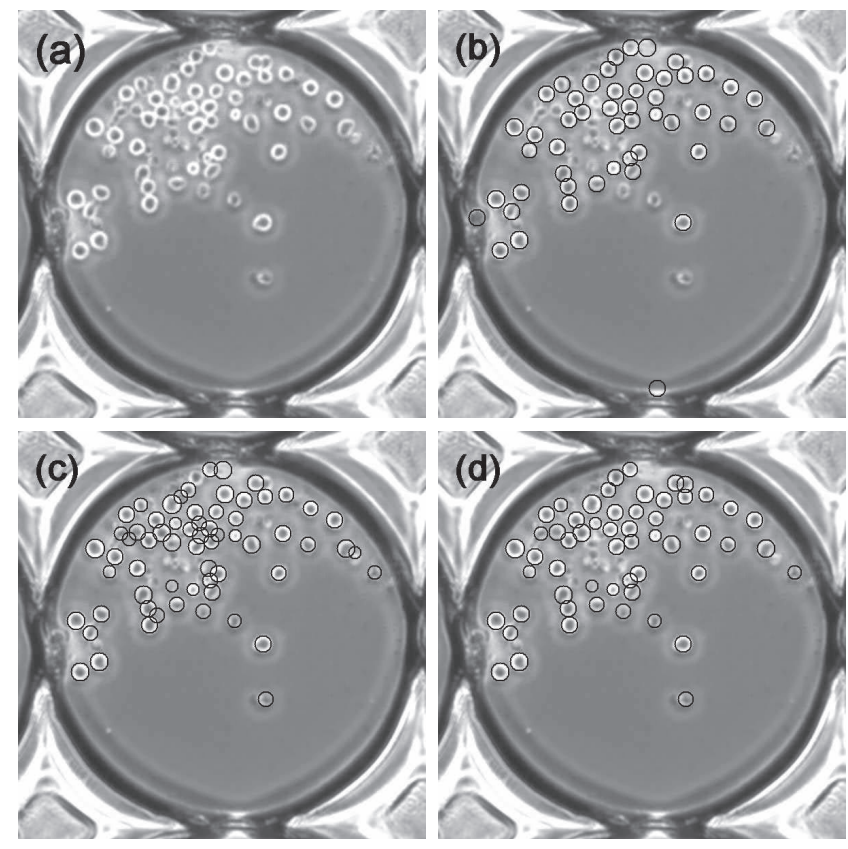

Fig. 6. Comparison of detection results. (a) Input image. (bd) Detection results using Hough transform, correlation, and the proposed method, respectively.

As presented in Table 1, the proposed method that utilized the output pattern of a ring filter bank achieved 96.5\% precision and $94.4 \%$ recall. Inferior detection performance was obtained for the standard methods. In particular, we observed that Hough transform is sensitive to the variations of shape and appearance which was reflected by low recall (high false negatives), while correlation generates high false positives due to touching and overlapping cells. Our proposed method yielded better robustness in both precision and recall, and led to better detection accuracy. Fig. 6 shows the comparison of detection results.

\section{CONCLUSION}

We proposed an accurate and robust method for detecting hematopoietic stem cells in phase contrast microscopy images, which exploits the output pattern of a multiple-radius ring filter bank. Compared to the standard detection schemes, our approach improved detection accuracy. In addition to
HSC detection, our method is potentially useful for identifying its morphological changes and mitosis based on a fitted quadratic model. It is also generally applicable to detecting other structures with variable scales.

\section{ACKNOWLEDGEMENTS}

We are grateful to E. D. Ker, Drs. E. D. Miller, P. G. Campbell, and L. E. Weiss for cell culture and imaging. We would also like to thank Drs. K. Li, M. Chen, and Z. Yin who gave useful comments. This work was supported in part by the National Institutes of Health Grants R01 EB07369-01 and R01 EB04343-01, and the Korea Research Foundation Grant KRF-2007-357-D00194.

\section{REFERENCES}

[1] National Institutes of Health, Stem Cells: Scientific Progress and Future Research Directions, Department of Health and Human Services, June 2001.

[2] T. J. Atherton and D. J. Kerbyson, "Size invariant circle detection," Image and Vision Computing, vol. 17, pp. 795-803, 1999.

[3] M. Smereka and I. Duleba, "Circular object detection using a modified hough transform," Int. J. Appl. Math. Comput. Sci., vol. 18, no. 1, pp. 85-91, 2008.

[4] T. Peng, A. Balijepalli, S. K. Gupta, and T. LeBrun, "Algorithms for on-line monitoring of micro spheres in an optical tweezers-based assembly cell," Transactions of the ASME, vol. 7, pp. 330-338, 2007.

[5] B. Kumar, A. Mahalanobis, and R. D. Juday, Correlation Pattern Recognition, Cambridge University Press, 2005.

[6] L. Miroslaw, A. Chorazyczewski, F. Buchholz, and R. Kittler, "Correlation-based method for automatic mitotic cell detection in phase contrast microscopy," in Proc. Int. Conf. Computer Recognition Systems, 2005, pp. 627-634.

[7] N. N. Kachouie, L. J. Lee, and P. Fieguth, "A probabilistic living cell segmentation model," in Proc. IEEE ICIP, 2005, pp. 901-904.

[8] N. N. Kachouie, P. Fieguth, J. Ramunas, and E. Jervis, "Probabilistic model-based cell tracking," Int. J. Biomed. Imag., vol. 2006, pp. 1-10, 2006. 